\title{
The Results of Conversion Therapy for Initially Unresectable Gastric Cancer: a Respective Analysis of 191 Patients
}

\section{Shaopeng Zhang}

First Hospital of Jilin University

\section{Song Wu}

First Hospital of Jilin University

\section{Yuan Kong}

First Hospital of Jilin University

Wei Li ( $\nabla$ weili8308@jlu.edu.cn )

First Hospital of Jilin University

\section{Research}

Keywords: Conversion Therapy , Gastric Cancer, chemotherapy

Posted Date: August 23rd, 2021

DOI: https://doi.org/10.21203/rs.3.rs-818857/v1

License: (c) (i) This work is licensed under a Creative Commons Attribution 4.0 International License. Read Full License 
The Results of Conversion Therapy for Initially Unresectable Gastric Cancer: a Respective Analysis of 191 Patients

\section{Abstract:}

【Objective】 To analyze the clinical efficacy of systemic chemotherapy combined with intraperitoneal hyperthermic perfusion in the treatment of locally invasive stage III gastric cancer and stage IV gastric cancer.

【Methods】 The clinical data of 191 patients with gastric cancer who received systemic chemotherapy combined with intraperitoneal hyperthermic perfusion from June 2010 to December 2018 were retrospectively analyzed.

【Results】 The unresectable factors in 191 patients with gastric cancer included peritoneum metastasis (106), local invasion (67), liver metastasis (25), lung metastasis (3), bone metastasis (4), adnexal metastasis (3) and adrenal metastasis (4). After conversion therapy, 191 patients were divided into finished conversion group and non-finished group. There were significant differences in T stage, M stage and tumor differentiation between the two groups. During the course of chemotherapy, 11 patients had grade 3 or 4 chemotherapy adverse reactions. The median survival was 36 months in the finished conversion group and 14 months in the non-finished group. The median survival of the 69 R0 resected patients was 38 months, which was higher than that of chemotherapy alone (14 months), best supportive care (13 months) and patients who completed chemotherapy without R0 resection (19 months). Univariate Cox survival analysis found that $\mathrm{N}$ stage, R0 resection, response to chemotherapy and unresectable factors were prognostic factors. Multivariate Cox survival analysis showed that $\mathrm{N}$-stage, response to chemotherapy and unresectable factors were independent prognostic factors.

【Conclusion】 For unresectable gastric cancer patients, surgical treatment after chemotherapy can prolong survival. Radical surgical treatment after conversion therapy and chemotherapy response are important factors related to patient survival. Chemotherapy alone can prolong survival in primary unresectable gastric cancer, but with limited effect.

\section{Background}

Gastric cancer is one of the most common malignant tumors in the world. The overall incidence rate has dropped dramatically (1) in the past 20 years, many surgical and medical strategies for gastric cancer have also been developing. However, the treatment of gastric cancer is still a challenge worldwide. Early radical resection can significantly improve the outcome of local gastric cancer, and many patients have long-term disease-free survival $(2,3)$. However, due to the occult onset of gastric cancer, many patients have been found with peritoneal metastasis, distant organ metastasis and other unresectable factors, and lost the opportunity of radical surgery, which makes the follow-up treatment extremely difficult (4). Chemotherapy is still the main treatment for these unresectable gastric cancer (5). Simple palliative resection and bypass surgery are commonly used in the treatment of gastric cancer caused by complications such as 
obstruction, hemorrhage or perforation $(6,7)$. In recent years, as a new treatment strategy, Conversion therapy for gastric cancer has attracted extensive attention. Conversion therapy uses radiotherapy, chemotherapy, targeted therapies to transform the initial unresectable tumor into radical resection, so as to prolong the survival time and improve the quality of life of patients (8, 9). At present, chemotherapy, radiotherapy, targeted therapy, palliative surgery and best supportive care have been widely used in the treatment of patients with advanced gastric cancer (10). Tsunematsum (11) performed 18 chemotherapy courses of S-1 plus cisplatin on a patient with gastric cancer with multiple liver metastases. After that, the liver metastases regress significantly, and then underwent open total gastrectomy and D2 1ymph node dissection. There was no recurrence 33 months after operation. Two randomized phase III clinical trials showed that the combination of surgery and chemotherapy can improve the prognosis of patients with advanced gastric cancer (12). A study analyzed the clinical data of 259 patients with stage IV gastric cancer who received systemic chemotherapy. 84 patients underwent surgery after chemotherapy, with a median survival of 41.3 months for $\mathrm{R} 0$ resected patients and 21.2 months for $\mathrm{R} 1-2$ resected patients, while the median survival of chemotherapy alone patients was only 11.3 months(13). At present, there are still some patients with gastric cancer undergoing conversion therapy who are unable to complete R0 resection due to chemotherapy insensitivity, intolerance and other factors. Kinoshita J(14) retrospectively studied the efficacy of combined chemotherapy with docetaxe1, cisplatin and S-1 (DCS) in stage IV gastric cancer and found that 34 of 57 patients completed surgical resection in the conversion therapy. Yasushi Sato(15) used docetaxel, cisplatin and S-1 (DCS) to treat 100 patients with unresectable gastric cancer, and finally achieved R0 resection in 28 patients. Currently, there is a lack of multicenter, prospective clinical data on translational therapy.

In our study, we analyzed the efficacy and feasibility of systemic chemotherapy combined with intraperitoneal hyperthermic perfusion in the treatment of stage IV gastric cancer and locally invasive stage III gastric cancer, and analyzed the clinicopathological factors related to prognosis.

\section{Patients and methods}

\section{1 Patients}

This study retrospectively analyzed 191 patients with stage IV and stage III gastric cancer with local invasion from the Department of gastrointestinal surgery, the First Bethune Hospital of Jilin University from June 2010 to December 2018. All patients received SOX+HIPEC. All patients were diagnosed as stage IV gastric cancer or locally invaded stage III gastric cancer by pathological examination, abdominal imaging examination or laparoscopic exploration, excluding patients with severe other organic diseases. All patients and their 
families signed informed consent before conversion therapy, which was approved by the Ethics Committee of the First Bethune Hospital of Jilin University. Inclusion criteria: (1) The patients came from the First Bethune Hospital of Jilin University, and the pathological definitive diagnosis was gastric cancer; (2) age less than 75 years; (3) signed informed consent; (4) The tumors were all primary and were diagnosed as stage IV gastric cancer or locally invasive stage III gastric cancer by abdominal imaging examination or laparoscopic exploration. (5) Bone marrow function, liver function, heart function and renal function are in good condition; (6) No other severe immunosuppressive diseases or concurrent malignancies. Exclusion criteria: (1) People with congenital diseases; (2) those with poor general status and severe organ lesions; (3) Previous history of radical or palliative surgery, radiotherapy and chemotherapy and biotherapy; (4) Complicated with gastrointestinal bleeding, perforation and serious infection.

All patients were given SOX+HIPEC. On the first day of conversion therapy, Oxaliplatin $(130 \mathrm{mg} / \mathrm{m} 2)$ was given intravenously for $2 \mathrm{~h}$ according to the patient's body surface area. On the 1st to 14th days of conversion therapy, S1 was taken orally at 40-60mg 2/ day. In each course of conversion therapy, intraperitoneal hyperthermic perfusion chemotherapy was given at the same time: $50 \mathrm{mg} / \mathrm{m} 2$ of cisplatin, $2000 \mathrm{~mL}$ of $0.9 \%$ sodium chloride solution was added, the perfusion speed was $35-45 \mathrm{~mL} / \mathrm{min}$, the water temperature was at $41^{\circ} \mathrm{C}$, and the infusion time was 45-60 min. Three weeks was a course, and the chemotherapy response is evaluated after two courses of treatment. The abdominal enhancement CT is used to evaluate the chemotherapy response based on Response Evaluation Criteria in Solid Tumors (RECIST) (16). Complete Response (CR) : the tumor disappeared completely; Partial response (PR) : the sum of the maximum diameter of the lesion decreased by $\geqslant 30 \%$; Stable disease $(\mathrm{SD})$ : the reduction degree of the sum of the maximum diameter of the lesion is between CR and PD. Progressive disease (PD) : the sum of the maximum diameter of the lesion increases by $\geqslant 20 \%$, or new lesions appear. After each chemotherapy, adverse reaction assessment was conducted according to WHO grading criteria for chemotherapy adverse reactions.

After completing 4 courses of conversion therapy, the patient was evaluated by abdominal CT and surgical exploration again, and then the patient was given 
surgical treatment, chemotherapy alone or the best supportive care. When preoperative evaluation indicated that radical surgery was possible, surgical treatment was given. After surgical treatment, the patient continued to receive adjuvant chemotherapy with SOX. However, some patients failed to receive surgical treatment due to the intolerance of chemotherapy, patient rejection, tumor progression and other related factors, and were then given chemotherapy alone or the best supportive care.

\section{2 statistical methods}

We used a chi-square test to analyze the general characteristics of the two groups of patients. Kaplan-Meier method was used to calculate survival time, and log-rank test was used to compare survival rate. Survival was calculated from the date of chemotherapy initiation to the date of death or most recent followup. Cox regression was performed to find the independent prognostic factors. All statistical analyses were performed using the SPSS 17.0 package. P value of 0.05 (bilateral) was defined as a statistically significant difference.

3. Results

3. 1 The conversion therapy results and the clinicopathological characteristics between finished conversion group and non-finished group

The conversion therapy results are shown in Figure 1. A total of 191 patients participated in this study. Among them, 86 patients received surgery after chemotherapy, among them there were 69 cases (80.2\%) received radical surgery, 11 cases (12.8\%) received palliative resection, and 6 cases (7\%) received gastrointestinal anastomosis. 105 patients were unable to accept surgical treatment because of intolerable chemotherapy adverse reaction, patient rejection, tumor progression and other related factors. Among the 105 patients, there were 56 cases (53.3\%) received standard chemotherapy, and 49 cases (46.7\%) received The best supportive care after only a few courses of chemotherapy.-

The clinicopathological characteristics and chemotherapy results of patients between finished conversion group and non-finished group are shown in Table 1. The differences in $\mathrm{T}$ stage, M stage, chemotherapy response, adverse reactions and histologic differentiation between the two groups were statistically significant. Among 191 patients, 78 patients had PR, 15 patients had PD, 72 patients had SD, and 26 patients who could not be evaluated. The finished conversion group included $46 \mathrm{PR}$ patients, $46 \mathrm{SD}$ patients and $5 \mathrm{PD}$ patients. The non-finished group included 32 PR patients, 10 SD patients, 37 PD patients and 26 patients who could not be evaluated. The overall response rate was $40.8 \%$. There was a statistically significant difference in the response to chemotherapy between the two groups $(P<0.001)$. Leucopenia (50 cases), thrombocytopenia (31 cases) and nausea and vomiting (29 cases) were the most common adverse reactions of chemotherapy. Only 3 patients had elevated transaminase and 5 patients had 
creatinine elevation. The incidence of adverse reactions was higher in the finished conversion group than in the non-finished group $(\mathrm{P}<0.001)$. There were no statistically significant differences in age, gender, $\mathrm{N}$ stage, Borrmann type and tumor location between the two groups.

\section{2 Unresectable factors of gastric cancer}

The unresectable factors are shown in Table 2, including 106 cases of peritoneum metastasis $(55.5 \%), 67$ cases of local invasion (35.1\%), 25 cases of liver metastasis (13.1\%), 3 cases of lung metastasis (1.6\%), 4 cases of bone metastasis (2.1\%), 3 cases of accessory metastasis (1.6\%) and 4 cases of adrenal metastasis (2. 1\%). Peritoneum metastasis is the most common unresectable factor in patients with gastric cancer. There was significant difference in the number of patients with liver metastasis between finished conversion group and nonfinished group $(P=0.02)$, and there was no significant statistically difference in other unresectable factors between the two groups. The number of patients with 2 or more unresectable factors in the finished conversion group (5 cases) was significantly lower than that in the non-finished group (26 cases), and the difference was statistically significant $(\mathrm{P}<0.001)$.

3. 3 Data of patients in the finished conversion group

86 patients in the finished conversion group are shown in Table 3 . No perioperative death occurred in all patients. Among the 86 finished conversion therapy patients, 69 patients $(80.2 \%)$ underwent R0 resection (including 30 distal gastrectomy, 36 total gastrectomy and 3 proximal gastrectomy). 17 patients (19.8\%) underwent R1 / R2 surgery (11 patients underwent palliative resection due to peritoneal metastasis, local invasion and other factors, and 6 patients underwent simple exploration or gastrointestinal anastomosis).16 patients (18.6\%) had perioperative complications, including 3 cases of hemorrhage, 6 cases of pneumonia, 5 cases of gastrointestinal fistula, 1 case of postoperative ileus, 1 case of abdominal infection.

\section{4 Survival Analysis}

Figure 2 shows the survival of patients in this study. The median survival of 86 patients in finished conversion group was 36 months, which was higher than that the 14 months of 105 patients in non-finished group ( $<<0.001$ ) (Fig. 2a). The median survival of patients with R0 resection was 38 months, which was higher than that the 19 months of patients with R1 and R2 resection. But the median survival time of $\mathrm{R} 1$ and $\mathrm{R} 2$ resection group was higher than that of non-finished group. The median survival time of patients with chemotherapy alone and best supportive treatment was 14 months, and there was no significant difference between the two groups (Fig. 2b). Table 4 shows the results of Univariate COX Regression Analysis of survival. N stage, R0 resection, response to chemotherapy and unresectable factors are prognostic factors related to survival. Table 5 shows the results of multivariate COX regression analysis. $N$ stage, response to chemotherapy and unresectable factors are independent prognostic factors related 
Discuss

Gastric cancer remains to be a major obstacle to global health. So far, a large number of studies have shown that preoperative adjuvant chemotherapy is the preferred alternative treatment for advanced gastric cancer or stage IV gastric cancer with peritoneal metastasis(17-19). The guidelines of NCCN also recommend preoperative adjuvant chemotherapy as the first choice for the advanced gastric cancer(20). There are also a large number of research which prove that the adjuvant chemotherapy can improve both R0 resection rate and overall survival, without affecting the incidence of perioperative complications(21, 22). Preoperative adjuvant chemotherapy is used to increase R0 resection by downstaging the tumor and eliminating occult metastasis(23). At present, the clinical efficacy evaluation of chemotherapy mainly includes molecular marker analysis, molecular imaging evaluation, anatomical imaging evaluation and histopathological response evaluation(24-27). The anatomical imaging evaluation and histopathological response evaluation are commonly used to evaluate tumor regression after chemotherapy. In this study, the response of chemotherapy was evaluated after 2 courses of chemotherapy. Some patients were evaluated as SD or PD by imaging, but the peritoneal implantation metastasis disappear during laparoscopic exploration. And so, further radical surgical resection was performed to prolong the survival period. The anatomical imaging evaluation can' $t$ fully reflect the response to chemotherapy, especially in patients with gastric cancer with peritoneal metastasis. The laparoscopic exploration is also necessary to patients with gastric cancer with peritoneal metastasis.

The unresectable factors of gastric cancer consist mainly of peritoneal metastasis, liver metastasis, local invasion, lung metastasis, bone metastasis, etc. Among all the possible factors, peritoneal metastasis is the most common in stage IV gastric cancer. Peritoneal metastasis occurs in $14 \%$ to $43 \%$ of patients with gastric cancer, and accounts for 35\% of all distant metastases (28, 29). In recent years, peritoneal hyperthermia therapy for peritoneal metastasis has been gradually applied(30). A phase randomized trial results consistently show that surgery combined with hyperthermia therapy can improve survival rate compared with surgery alone $(31,32)$. In our study, there were totally 106 patients unable to receive primary radical surgery due to peritoneal metastasis. While after preoperative chemotherapy, 45 patients (42.4\%) received surgical treatment to prolong their survival. Liver metastasis is the first stop of blood metastasis of gastric cancer. Japan guidelines recommend systemic chemotherapy as the standard treatment of hepatic metastatic gastric cancer patients(33). Yasushi Sato(15) performed transformation therapy on 100 patients with unresectable gastric cancer. There are 29 patients with liver metastasis in 100 patients, only 6 received surgical treatment after completion of transformation therapy. 
In this study, 25 of the 191 patients had liver metastases, of whom 6 underwent surgery after conversion therapy. We found that patients with liver metastasis in the finished conversion group were significantly lower than those in the nonfinished group, and the sensitivity of patients with liver metastasis to this experimental chemotherapy regimen was low. So the optimal chemotherapy regimen for patients with gastric cancer combined with liver metastases still needs further study.

At present, chemotherapy drugs for stage IV gastric cancer mainly include S1, cisplatin, paclitaxel, irinotecan, fluorouracil and oxaliplatin (34-36). In this study, patients were treated with systemic intravenous oxaliplatin, S1 (DCS) combined with cisplatin intraperitoneal hyperthermic perfusion. After preoperative chemotherapy, $45(42.5 \%)$ of 106 patients with peritoneal metastatic lesions disappeared, and then received radical surgical treatment. Among 191 patients, 69 patients (36.1\%) received radical surgical treatment, which was generally higher than that of patients with systemic chemotherapy alone. Some clinical studies have shown that $20 \%$ of patients with unresectable gastric cancer can achieve R0 resection after conversion therapy $(13,37)$. Results of a phase III randomized clinical trial showed that intraperitoneal hyperthermia improved the prognosis of patients with peritoneal metastatic gastric cancer (38). However, for gastric cancer patients with liver metastasis, lung metastasis and other hemorrhagic metastases, the effect of intraperitoneal hyperthermia therapy is relatively poor. In this study, there were 25 patients with liver metastasis, and 4 patients (18\%) received radical surgical treatment after chemotherapy. A more effective conversion chemotherapy for patients with hemorrhagic metastatic gastric cancer needs to be further explored.

A growing number of scholars have advocated conversion therapy for IV gastric cancer and have found that completion of R0 resection after successful conversion therapy significantly prolongs patient survival (34, 36, 37, 39). The current study found that the median survival for completed R0 resection was 19-62 months, while the median survival for failure to undergo R0 resection was 8-14 months. In our study, the median survival was 38 months for patients who underwent R0 resection, compared to about 14 months for patients who received chemotherapy alone and best supportive care, which is similar to previous studies. 45\% of patients in this study received surgery after chemotherapy, while the remaining 55 percent with poor prognosis received chemotherapy alone or best supportive care. How to improve the success rate of conversion therapy is a problem at present. The potential biomarkers related to chemotherapy sensitivity of gastric cancer have been found by a large number of studies. Fujimoto (40) detected the expression of RPN2 in 40 patients with advanced gastric cancer who received 
docetaxel combined with cisplatin preoperative chemotherapy, and found that the clinicopathological response of the RPN2-negative group to docetaxel combined with cisplatin is better than that of the RPN2-positive group. The expression of RPN2 in upper gastrointestinal biopsy tissue can be used as a predictive biomarker for the efficacy of docetaxel and cisplatin in advanced gastric cancer. A metaanalysis study found that glutathione-S-transferase p1 gene expression was significantly associated with chemotherapy sensitivity in patients with gastric and rectal cancer, while no significant association was found with chemotherapy toxicity (27). Our study found that there was statistically significance in differences between the finished conversion group and the non-finished group in T stage, M stage, histologic differentiation, response to chemotherapy efficacy and unresectable factors. $\mathrm{N}$ stage, response to chemotherapy and unresectable factors are independent factors related to prognostic factors.

\section{Conclusion}

For patients with unresectable gastric cancer, sox combined with cisplatin intraperitoneal thermal perfusion therapy can achieve R0 resection and prolong survival in some patients. The efficacy of this chemotherapy for peritoneal metastatic cancer is better than that for gastric cancer patients with blood metastasis. Radical surgical treatment after conversion therapy, chemotherapy response, $\mathrm{N}$-stage and unresectable factors are important factors associated with patient survival. $\mathrm{N}$-stage, response to chemotherapy and unresectable factors are independent factors associated with prognosis. Laparoscopic exploration is an important method for assessing the efficacy of chemotherapy in patients with gastric cancer with peritoneal metastases. Chemotherapy alone can prolong the survival of primary unresectable gastric cancer alone, the effect is limited.

Figure 1 Flow diagram of the patients' clinical course

\section{Figure 2}

A:Survival analysis based on status of conversion therapy of all the 191 patients. B:Survival analysis between radical surgery group, non-radical group, chemotherapy group and BSC group. 
1. Ferlay J, Shin HR, Bray F, Forman D, Mathers C and Parkin DM: Estimates of worldwide burden of cancer in 2008: GLOBOCAN 2008. International journal of cancer 127: 2893-2917, 2010.

2. Alberts SR, Cervantes A and van de Velde CJ: Gastric cancer: epidemiology, pathology and treatment. Annals of oncology : official journal of the European Society for Medical Oncology 14 Suppl 2: ii31-36, 2003.

3. Saka M, Morita S, Fukagawa T and Katai H: Present and future status of gastric cancer surgery. Japanese journal of clinical oncology 41: 307-313, 2011.

4. Shah MA: Update on metastatic gastric and esophageal cancers. Journal of clinical oncology : official journal of the American Society of Clinical Oncology 33: 1760-1769, 2015.

5. Sano T and Aiko T: New Japanese classifications and treatment guidelines for gastric cancer: revision concepts and major revised points. Gastric cancer : official journal of the International Gastric Cancer Association and the Japanese Gastric Cancer Association 14: 97-100, 2011.

6. Shen L, Shan YS, Hu HM, et al.: Management of gastric cancer in Asia: resource-stratified guidelines. The Lancet. Oncology 14: e535-547, 2013.

7. Mahar AL, Coburn NG, Karanicolas PJ, Viola R and Helyer LK: Effective palliation and quality of life outcomes in studies of surgery for advanced, non-curative gastric cancer: a systematic review. Gastric cancer : official journal of the International Gastric Cancer Association and the Japanese Gastric Cancer Association 15 Suppl 1: S138-145, 2012.

8. Yoshida K, Yamaguchi K, Okumura N, Tanahashi T and Kodera Y: Is conversion therapy possible in stage IV gastric cancer: the proposal of new biological categories of classification. Gastric cancer : official journal of the International Gastric Cancer Association and the Japanese Gastric Cancer Association 19: 329-338, 2016.

9. Wang $\mathrm{T}$, Wang $\mathrm{N}$, Ren $\mathrm{H}$, et al.: Long-term Results of Conversion Therapy for Initially Unresectable Gastric Cancer: Analysis of 122 Patients at the National Cancer Center in China. Journal of Cancer 10: 5975-5985, 2019.

10. Izuishi K and Mori H: Recent Strategies for Treating Stage IV Gastric Cancer: Roles of Palliative Gastrectomy, Chemotherapy, and Radiotherapy. Journal of gastrointestinal and liver diseases : JGLD 25: 87-94, 2016.

11. Tsunematsu M, Takahashi N, Murakami K, Misawa T, Akiba T and Yanaga K: Successful conversion surgery for gastric cancer with multiple liver metastases treated after $S-1$ plus cisplatin combination chemotherapy: a case report. Surgical case reports 3: 95, 2017.

12. Ychou M, Boige V, Pignon JP, et al.: Perioperative chemotherapy compared with surgery alone for resectable gastroesophageal adenocarcinoma: an FNCLCC and FFCD multicenter phase III trial. Journal of clinical oncology : official journal of the American Society of Clinical Oncology 29: 1715 1721, 2011.

13. Yamaguchi $\mathrm{K}$, Yoshida $\mathrm{K}$, Tanahashi $\mathrm{T}$, et al.: The long-term survival of stage IV gastric cancer patients with conversion therapy. Gastric cancer : official journal of the International Gastric Cancer Association and the Japanese Gastric Cancer Association 21: 315-323, 2018.

14. Kinoshita J, Fushida S, Tsukada T, et al.: Efficacy of conversion gastrectomy following 
docetaxel, cisplatin, and S-1 therapy in potentially resectable stage IV gastric cancer. European journal of surgical oncology : the journal of the European Society of Surgical Oncology and the British Association of Surgical Oncology 41: 1354-1360, 2015.

15. Sato $\mathrm{Y}$, Ohnuma H, Nobuoka $\mathrm{T}$, et al.: Conversion therapy for inoperable advanced gastric cancer patients by docetaxel, cisplatin, and S-1 (DCS) chemotherapy: a multi-institutional retrospective study. Gastric cancer : official journal of the International Gastric Cancer Association and the Japanese Gastric Cancer Association 20: 517-526, 2017.

16. Eisenhauer EA, Therasse $P$, Bogaerts J, et al.: New response evaluation criteria in solid tumours: revised RECIST guideline (version 1.1). European journal of cancer (Oxford, England : 1990) 45: 228-247, 2009

17. Sato Y, Sagawa T, Ohnuma H, et al.: A dose-escalation study of docetaxel, oxaliplatin, and S1 (DOS) as a first-line therapy for patients with unresectable metastatic gastric cancer. Cancer chemotherapy and pharmacology 83: 161-167, 2019.

18. Gong Y, Wang P, Zhu Z, et al.: Benefits of Surgery After Neoadjuvant Intraperitoneal and Systemic Chemotherapy for Gastric Cancer Patients With Peritoneal Metastasis: A Meta-Analysis. The Journal of surgical research 245: 234-243, 2020.

19. Du R, Hu P, Liu Q and Zhang J: Conversion Surgery for Unresectable Advanced Gastric Cancer: A Systematic Review and Meta-Analysis. Cancer investigation 37: 16-28, 2019.

20. Ajani JA, Bentrem DJ, Besh S, et al.: Gastric cancer, version 2.2013: featured updates to the NCCN Guidelines. Journal of the National Comprehensive Cancer Network : JNCCN 11: 531 -546, 2013.

21. Ge L, Wang HJ, Yin D, et al.: Effectiveness of 5-flurouracil-based neoadjuvant chemotherapy in locally-advanced gastric/gastroesophageal cancer: a meta-analysis. World journal of gastroenterology 18: 7384-7393, 2012.

22. Sym SJ, Chang HM, Ryu MH, et al.: Neoadjuvant docetaxel, capecitabine and cisplatin (DXP) in patients with unresectable locally advanced or metastatic gastric cancer. Annals of surgical oncology 17: 1024-1032, 2010.

23. Cho H, Nakamura J, Asaumi Y, et al.: Long-term survival outcomes of advanced gastric cancer patients who achieved a pathological complete response with neoadjuvant chemotherapy: a systematic review of the literature. Annals of surgical oncology 22: 787-792, 2015.

24. Ang J, Hu L, Huang PT, et al.: Contrast-enhanced ultrasonography assessment of gastric cancer response to neoadjuvant chemotherapy. World journal of gastroenterology 18: 7026-7032 2012.

25. Kurokawa $Y$, Shibata $T$, Sasako $M$, et al.: Validity of response assessment criteria in neoadjuvant chemotherapy for gastric cancer (JCOG0507-A). Gastric cancer : official journal of the International Gastric Cancer Association and the Japanese Gastric Cancer Association 17: 514-521, 2014.

26. Heger U, Bader F, Lordick F, et al.: Interim endoscopy results during neoadjuvant therapy for gastric cancer correlate with histopathological response and prognosis. Gastric cancer : official journal of the International Gastric Cancer Association and the Japanese Gastric Cancer Association 17: 478-488, 2014.

27. Shen X, Wang J, Yan X, et al.: Predictive value of GSTP1 lle105Val polymorphism in clinical outcomes of chemotherapy in gastric and colorectal cancers: a systematic review and metaanalysis. Cancer chemotherapy and pharmacology 77: 1285-1302, 2016. 
28. Thomassen I, van Gestel YR, van Ramshorst B, et al.: Peritoneal carcinomatosis of gastric origin: a population-based study on incidence, survival and risk factors. International journal of cancer 134: 622-628, 2014.

29. Abbasi SY, Taani HE, Saad A, Badheeb A and Addasi A: Advanced gastric cancer in jordan from 2004 to 2008: a study of epidemiology and outcomes. Gastrointestinal cancer research : GCR 4: 122-127, 2011.

30. Ramos M, Pereira MA, Charruf AZ, et al.: CONVERSION THERAPY FOR GASTRIC CANCER: EXPANDING THE TREATMENT POSSIBILITIES. Arquivos brasileiros de cirurgia digestiva : $A B C D=$ Brazilian archives of digestive surgery 32: e1435, 2019.

31. Yang XJ, Huang CQ, Suo T, et al.: Cytoreductive surgery and hyperthermic intraperitoneal chemotherapy improves survival of patients with peritoneal carcinomatosis from gastric cancer: final results of a phase III randomized clinical trial. Annals of surgical oncology 18: 1575-1581, 2011.

32. Rudloff U, Langan RC, Mullinax JE, et al.: Impact of maximal cytoreductive surgery plus regional heated intraperitoneal chemotherapy (HIPEC) on outcome of patients with peritoneal carcinomatosis of gastric origin: results of the GYMSSA trial. Journal of surgical oncology 110: 275 284, 2014.

33. Japanese gastric cancer treatment guidelines 2010 (ver. 3). Gastric cancer : official journal of the International Gastric Cancer Association and the Japanese Gastric Cancer Association 14: 113 123, 2011.

34. Satoh S, Okabe H, Teramukai S, et al.: Phase II trial of combined treatment consisting of preoperative S-1 plus cisplatin followed by gastrectomy and postoperative S-1 for stage IV gastric cancer. Gastric cancer : official journal of the International Gastric Cancer Association and the Japanese Gastric Cancer Association 15: 61-69, 2012.

35. Kanda T, Yajima K, Kosugi S, Ishikawa T, Ajioka Y and Hatakeyama K: Gastrectomy as a secondary surgery for stage IV gastric cancer patients who underwent S-1-based chemotherapy: a multi-institute retrospective study. Gastric cancer : official journal of the International Gastric Cancer Association and the Japanese Gastric Cancer Association 15: 235-244, 2012.

36. Morgagni P, Solaini L, Framarini M, et al.: Conversion surgery for gastric cancer: A cohort study from a western center. International journal of surgery (London, England) 53: 360 -365, 2018 37. Fukuchi M, Ishiguro T, Ogata K, et al.: Prognostic Role of Conversion Surgery for Unresectable Gastric Cancer. Annals of surgical oncology 22: 3618-3624, 2015.

38. Wang $P$ and Zhou ZX: Cytoreductive Surgery and Hyperthermic Intraperitoneal Chemotherapy Improves Survival of Patients with Peritoneal Carcinomatosis from Gastric Cancer: Trial Results of a Phase 2, Randomized, Clinical Trial. Annals of surgical oncology 24: 623, 2017.

39. Kim SW: The result of conversion surgery in gastric cancer patients with peritoneal seeding Journal of gastric cancer 14: 266-270, 2014.

40. Fujimoto D, Goi T, Koneri K and Hirono Y: RPN2 is effective biomarker to predict the outcome of combined chemotherapy docetaxel and cisplatin for advanced gastric cancer. Oncotarget 9 : 15208-15218, 2018. 
Figures



Figure 1

Flow diagram of the patients' clinical course

A:Survival analysis between Conversion( + ) and Conversion(-)

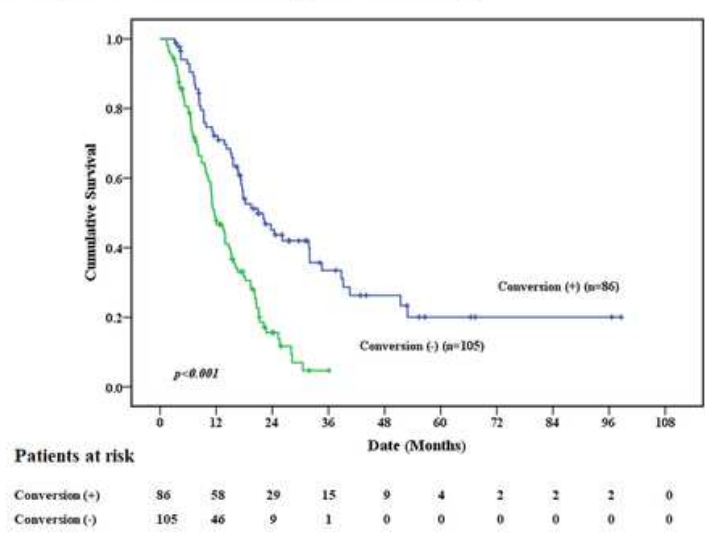

B:Survival analysis between Radical Surgery, Non-Radical Surgery, Chemotherapy and BSC

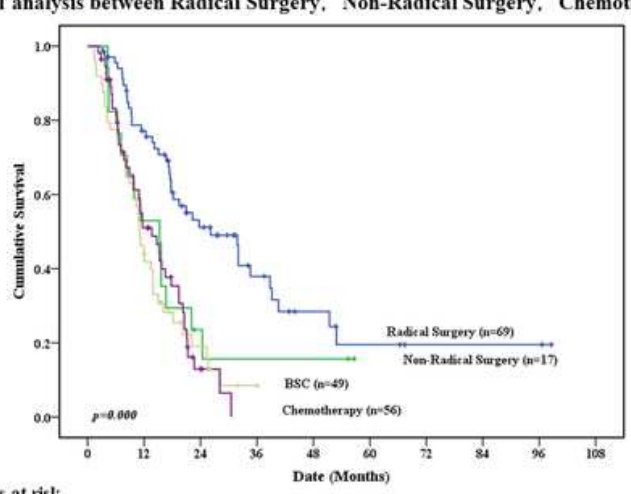

Patients at risk

Radical Surgery Chemotherapy BSC

Figure 2 
A:Survival analysis based on status of conversion therapy of all the 191 patients. B:Survival analysis between radical surgery group, non-radical group, chemotherapy group and BSC group. 